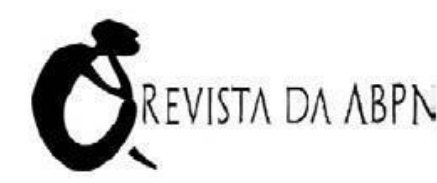

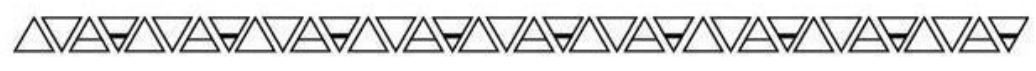

DOI 10.31418/2177-2770.2020.v12.c3.p138-151 | ISSN 2177-2770

Licenciado sob uma Licença Creative Commons

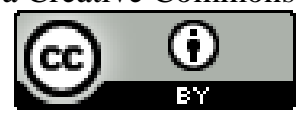

\section{POR UMA POLÍTICA DE SAÚDE MENTAL DA POPULAÇÃO} NEGRA NO SUS

\author{
Jeane Saskya Campos Tavares ${ }^{1}$ \\ Carlos Antonio Assis de Jesus Filho ${ }^{2}$ \\ Elisangela Ferreira de Santana ${ }^{3}$
}

Resumo: A implementação de políticas públicas direcionadas à saúde da população negra brasileira tem sido insuficiente e isto contribui para a manutenção da hierarquia racial estabelecida pelo colonialismo. Em relação à saúde mental, o modelo hospitalocêntrico, patologizante e centrado na medicalização, na eugenia e no racismo científico, foi hegemônico no início do século XX e se atualiza no século XXI através das Comunidades Terapêuticas e da reinserção de hospitais como elementos centrais da Rede de Atenção Psicossocial (RAPS). Vasta literatura internacional destaca o racismo como importante produtor de sofrimento persistente, com significativo prejuízo individual e coletivo. No entanto, este tem sido um tema negligenciado no contexto nacional, especialmente na Saúde Pública. A proposição e construção de uma política pública efetiva de promoção da saúde mental passa pela pactuação entre as diferentes esferas de governo de uma política de saúde mental racializada, pela superação do racismo institucional e pela radical ruptura com o modelo manicomial.

Palavras-chaves: População Negra; Saúde Mental; Racismo; Reforma psiquiátrica; Movimento antimanicomial.

\section{POR UMA POLÍTICA DE SAÚDE MENTAL DA POPULAÇÃO NEGRA NO SUS}

Abstract: The implementation of public policies aimed at the health of the brazilian black population has been insufficient and this contributes to the maintenance of the racial

\footnotetext{
${ }^{1}$ Psicóloga. Dra em Saúde Pública (ISC/UFBA). Docente da Universidade Federal do Recôncavo da Bahia (UFRB). ORCID: https://orcid.org/0000-0001-5745-1417. E-mail: jeanesctavares@ gmail.com

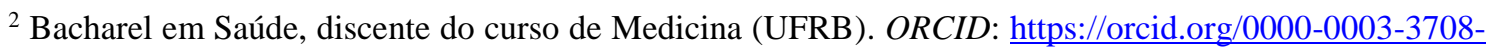
6568; E-mail: nino_assis@outlook.com

${ }^{3}$ Psicóloga, Especialista em Saúde da Família da Família (UFRB/ESPBA). ORCID: https://orcid.org/00000003-2132-8972; E-mail: eli.santana.psi@gmail.com
}

Revista da ABPN • v. 12, n. Ed. Especial - Caderno Temático: "III ANPSINEP Articulação Nacional de Psicólogas/os Negras/os e Pesquisadoras/es” • outubro de 2020, p. 138-151 


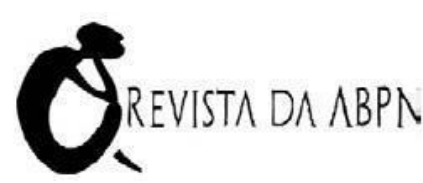

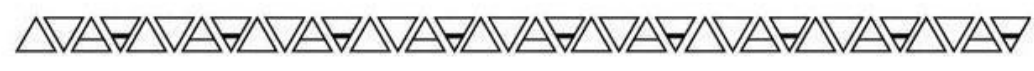

hierarchy established by colonialism. In relation to mental health, the hospital-centered, pathological and centered on medicalization, eugenics and scientific racism, was hegemonic in the beginning of the 20th century, and it is updated on the 21st century through the Therapeutic Communities and the reintegration of hospitals as central elements of the Network of Psychosocial Care (RAPS). Extensive international literature highlights racism as an important producer of persistent suffering, with significant individual and collective damage. However, this has been a neglected topic in the national context, especially in Public Health. The proposition and construction of an effective public policy for the promotion of mental health involves the agreement between the different spheres of government of a racialized mental health policy, the overcoming of institutional racism and the radical break with the asylum model.

Keywords: Black population; Mental health; Racism; Psychiatric reform; Anti-asylum movement

\section{POR UMA POLÍTICA DE SAÚDE MENTAL DA POPULAÇÃO NEGRA NO SUS}

Resumen: La implementación de políticas publicas direccionadas a la salud del pueblo afrodescendiente Brasileño ha sido insuficiente y eso contribuye para el mantenimiento de la jerarquía racial establecida por el colonialismo. Ya sobre la salud mental, el modelo "hispotalocentrico", patológico y centrado en la medicación, en la eugenesia y en el racismo científico, fue hegemónico en el inicio del siglo XX y se actualiza en el siglo XXI por las comunidades terapéuticas y de la reinserción de hospitales como elementos del centro de la red de atención psicosocial (RAPS). Vasta literatura internacional destaca el racismo como importante productor de sufrimiento persistente y colectivo. Sin embargo, ese ha sido un tema descuidado en el contexto nacional, específicamente en la salud pública. La proposición y construcción de una política pública efectiva de la valoración de la salud mental pasa por el acuerdo entre la diferentes esferas del gobierno de una política de salud mental racial, por la superación del racismo institucional y por la radical ruptura con el modelo del manicomio.

Palabras clave: Pueblo afrodescendiente; Salud mental; Racismo; Reforma psiquiátrica; Movimiento antimanicomial

\section{POR UMA POLÍTICA DE SAÚDE MENTAL DA POPULAÇÃO NEGRA NO SUS}

Resumé: La mise en œuvre de politiques publiques visant la santé de la population noire brésilienne a été insuffisante et cela contribue au maintien de la hiérarchie raciale établie par le colonialisme. En ce qui concerne la santé mentale, le modèle pathologique centré sur l'hôpital et centré sur la médicalisation, l'eugénisme et le racisme scientifique, était hégémonique au début du $20 \mathrm{e}$ siècle et est mis à jour au $21 \mathrm{e}$ siècle à travers les Communautés thérapeutiques et la réintégration des hôpitaux comme éléments centraux du Réseau des soins psychosociaux (RAPS). Une littérature internationale abondante met en évidence le racisme comme un important producteur de souffrances persistantes, avec

Revista da ABPN • v. 12, n. Ed. Especial - Caderno Temático: "III ANPSINEP Articulação Nacional de Psicólogas/os Negras/os e Pesquisadoras/es” • outubro de 2020, p. 138-151 
des dommages individuels et collectifs importants. Cependant, cela a été un sujet négligé dans le contexte national, en particulier en santé publique. La proposition et la construction d'une politique publique efficace pour la promotion de la santé mentale passe par l'accord entre les différentes sphères de gouvernement d'une politique de santé mentale racialisée, le dépassement du racisme institutionnel et la rupture radicale avec le modèle d'asile.

Mots clés: population noire; Santé mentale; Racisme; Réforme psychiatrique; Mouvement anti-asile.

\section{INTRODUÇÃO}

No Brasil, o campo da saúde mental teve seu início marcado pelo ideal eugenista, baseado no racismo científico e no medo branco (AZEVEDO, 1987) que caracterizaram o período entre o final do século XIX e início do século XX. Neste texto nos interessa que hospícios e manicômios tinham entre suas funções o controle da população negra recém liberta que, apesar de empobrecida e desamparada pelo Estado, era temida. Isto não apenas pelo número de pessoas negras ser significativamente maior que de brancos mas, principalmente, pela capacidade de organização e mobilização demonstrada nas fugas, nos ataques a plantações e engenhos, nos levantes e revoltas, na resistência nos quilombos, na greve de 1857 (REIS, 2019) e pelos resultados alcançados na Revolução Haitiana (AZEVEDO, 1987).

São inúmeras as publicações nacionais e internacionais que registram a história de terror e do uso de todo tipo de violência física, sexual, psicológica, moral e financeira contra encarcerados em hospícios e sanatórios sob alegação de oferta de tratamento médico durante a primeira metade do século XX. Não nos deteremos na exposição de métodos de controle e punição que caracterizavam o funcionamento destas instituições, nem mesmo nos benefícios trazidos à Medicina, tanto através de venda de corpos para as universidades quanto no desenvolvimento de uma percepção social de confiabilidade e cientificidade de seus métodos.

Embora a história registre proposições dissidentes como as de Juliano Moreira, a hegemonia do racismo científico, ou seja, a crença de que pessoas negras seriam essencialmente inclinadas à violência, à alienação (loucura), além de inferiores intelectual e moralmente contribuiu para que a psiquiatria, ao interpretar o sofrimento gerado pelas

Revista da ABPN • v. 12, n. Ed. Especial - Caderno Temático: "III ANPSINEP Articulação Nacional de Psicólogas/os Negras/os e Pesquisadoras/es” • outubro de 
péssimas condições sociais da época como patologias, reduzisse ao funcionamento individual os processos de adoecimento e "cura" nesta população. Esse deslocamento de responsabilidade da sociedade para o indivíduo fazia parte do esforço de apagamento da história de escravização e impedia a identificação do racismo como determinante na saúde física e mental da população negra.

Fazia parte, ainda, de um contexto de política de branqueamento adotada pelo Estado. Em meio ao fascismo e autoritarismo, o mito da democracia racial, se tornou um dos elementos fundantes da identidade do brasileiro. Tomava forma, o racismo à brasileira no qual, como afirma Schucman (2010), a crença na igualdade entre brancos e negros configurava-se como uma arma ideológica produzida por intelectuais das elites dominantes brancas, uma crença que protegia a sociedade racialmente hierarquizada por séculos de escravidão de conflitos raciais.

Temos em Lima Barreto um cronista que, como homem negro, vai narrar em primeira pessoa como o racismo era um importante mediador do encarceramento e um elemento fundamental na patologização do sofrimento psíquico decorrente da história de escravização e impedimento de acesso a diretos de cidadania. Em seu livro publicado postumamente, O Cemitério dos Vivos, o autor critica a "policialização" da saúde mental, sua aproximação com as ideias eugenistas e darwinistas ao promover o encarceramento de estrangeiros e pessoas de cor:

A polícia, não sei como e por quê, adquiriu a mania das generalizações, e as mais infantis. Suspeita de todo o sujeito estrangeiro com nome arrevesado. (...) todo cidadão de cor há de ser por força um malandro; e todos loucos hão de ser por força furiosos e só transponíveis em carros blindados" (BARRETO, 2017, p. 143, 144).

Apesar a riqueza de informações sobre este período, nos interessa destacar que, no pós Segunda Guerra, refutados todos os argumentos biológicos em que se pudesse basear o racismo científico, não se observou investimento financeiro ou político, proposições teóricas ou tomada de decisões relativas ao reconhecimento do racismo como elemento fundamental na produção de sofrimento psíquico na população negra. Os intelectuais e profissionais de saúde envolvidos no processo de ruptura com o modelo manicomial no Brasil, minimizaram ou mesmo desconsideram a questão racial no processo de patologização, encarceramento e morte nas instituições psiquiátricas.

Revista da ABPN • v. 12, n. Ed. Especial - Caderno Temático: "III ANPSINEP Articulação Nacional de Psicólogas/os Negras/os e Pesquisadoras/es” • outubro de 
Nos chama a atenção que a crença na igualdade racial está tão arraigada ao modo de funcionamento da sociedade brasileira que a história construída acerca da reforma psiquiátrica nacional ignora que Franco Basaglia foi fortemente influenciado por Frantz Fanon (PASSOS, 2018; SERAPIONI, 2019) que, por sua vez, considerava o colonialismo e o racismo aspectos centrais no processo de adoecimento mental da população negra. $\mathrm{Ou}$ seja, ainda que o principal ícone do movimento antimanicomial considerasse em sua obra o papel do racismo no adoecimento e encarceramento, isto não foi suficiente para que a militância por direitos humanos e desospitalização incluísse a pauta da luta antirracista.

Como afirma Passos (2018, p. 14), na experiência brasileira:

[...] não identificamos qualquer menção sobre a contribuição de Fanon para a Reforma Psiquiátrica e a Luta Antimanicomial, o que demonstra um afastamento do debate étnico-racial, em especial, sobre o colonialismo e seus efeitos sobre a constituição da subjetividade.

Além da ausência de menção à Fanon ou a qualquer relação entre encarceramento em manicômios, racismo e colonialismo, uma breve análise da produção de teóricos de referência em publicações brasileiras nos indica que a hierarquia racial foi mantida no movimento: homens brancos como intelectuais/profissionais de saúde ou gestores e pessoas negras como usuários do sistema e militantes orgânicos.

Referindo-se à invisibillização das contribuições de Fanon e ausência de discussão racial no movimento antimanicomial brasileiro, Passos (2018) convida o leitor a refletir sobre os motivos que nos levaram a comparar o genocídio nacional em instituições como o Hospital Colônia, na cidade de Barbacena (MG), com o holocausto nazista e não com nossa história de escravização e colonialismo, uma vez que estas categorias estruturam nossa sociedade e a subjetividade negra brasileira.

Neste ponto, especificamente, registramos alguma discordância que, embora ultrapasse o escopo deste texto, consideramos relevante. O modelo manicomial brasileiro traz em si tanto uma atualização das perversões coloniais - e, talvez por isso, seja aceito ou mesmo defendido por esta sociedade - quanto se aproxima dos crimes nazistas pela sistematização e pela função das mortes e torturas.

Na escravização a "peça" (corpo negro) tinha valor enquanto meio de produção. A tortura ou a morte pública e espetacular produziam o terror e, por fim, o controle dos 
escravizados. Na configuração dos manicômios brasileiros percebem-se tanto a manutenção desta lógica colonial de controle e exploração dos corpos negros, como a sistematização e o processo de aniquilamento progressivo dos campos de extermínio. Nesta configuração híbrida, o capital (em suas várias significações) é gerado pela exploração dos corpos dos prisioneiros para trabalhos forçados, pela possibilidade de submissão das pessoas vulnerabilizadas à tortura travestida de experimentos científicos e pela venda de cadáveres para os cursos de Medicina para produção das "peças" anatômicas.

Concordamos com Passos (2018) em relação ao reconhecimento de que a recusa em fazer o enfrentamento do racismo na saúde mental é como parte do funcionamento da sociedade brasileira. Baseando -se na discussão sobre racismo estrutural proposta por Almeida (2018), esta autora defende que:

A naturalização da violência institucional sobre os corpos negros compõe o modelo manicomial. O manicômio estrutura-se para além de uma edificação, pois mesmo com o fim do hospital psiquiátrico ainda experimentamos inúmeros formatos e formas de apartheid social, expressas pela via da medicalização e patologização da vida, pela internação compulsória, pela esterilização coercitiva, pela higienização urbana e demais fenômenos, ou seja, o manicômio é social, uma vez que ele está introjetado e reproduzido nas relações sociais (PASSOS, 2018, p.13).

A partir da análise empreendida por esta autora e por Davis (2018), compreendemos que a manutenção da violência e de instituições como prisões e manicômios na atualidade não é um acaso ou desconhecimento sobre as repercussões do encarceramento e tortura sobre a saúde mental da população negra. Antes a manutenção da lógica manicomial é parte do funcionamento de nossa sociedade e tem função importante no controle dos corpos negros e para a continuidade de complexos industriais de armas, tecnologia de segurança, produtos farmacêuticos, dentre outros.

\section{A SAÚDE MENTAL NA PNSIPN E NA RAPS}

Com esta breve introdução pretendemos destacar que o Estado brasileiro não é apenas omisso, negligente ou falho em relação à saúde da população negra. A oferta de

Revista da ABPN • v. 12, n. Ed. Especial - Caderno Temático: "III ANPSINEP Articulação Nacional de Psicólogas/os Negras/os e Pesquisadoras/es” • outubro de 
serviços de saúde subfinanciados ou desfinanciados, como já acontece com a EC 95 de 2016, é parte do racismo histórico e estrutural que se atualiza a cada governo produzindo vulnerabilidade e morte da população negra e indígena. Remetendo-nos à discussão proposta por Almeida (2018) é, portanto, parte do funcionamento normal de nossa sociedade.

Especificamente em relação à saúde mental, como destacamos anteriormente, as políticas públicas direcionadas a população negra têm em grande medida se baseado na patologização, medicalização, encarceramento e morte. E nem mesmo a reforma psiquiátrica/ movimento antimanicomial produziram tecnologias de cuidado racializadas e emancipadoras.

Embora o movimento negro venha apontando há décadas a conexão entre o racismo que organiza a sociedade brasileira, o sofrimento psíquico e a violência dos manicômios assim como a redução da discussão da saúde mental ao abuso de álcool e outras drogas, pouco avançamos no desenvolvimento teórico-prático de políticas em saúde mental que considerem as necessidades da população negra e que contribuam para o enfrentamento do racismo. Como exemplo, citamos a forte resistência à implementação da Política Nacional de Saúde Integral da População Negra (PNSIPN) que inclui como estratégias de gestão:

- Fortalecimento da atenção à saúde mental das crianças, adolescentes, jovens, adultos e idosos negros, com vistas à qualificação da atenção para o acompanhamento do crescimento, desenvolvimento e envelhecimento e a prevenção dos agravos decorrentes dos efeitos da discriminação racial e exclusão social.

- Fortalecimento da atenção à saúde mental de mulheres e homens negros, em especial aqueles com transtornos decorrentes do uso de álcool e outras drogas (BRASIL, 2013c).

A literatura nacional e internacional tem indicado as inúmeras repercussões do racismo na saúde mental de pessoas negras no âmbito individual e social/coletivo. Associam-se ao racismo elevados níveis de estresse, ansiedade, depressão, diminuição da aspiração pessoal, medos patológicos, retraimento social, dificuldade de auto cuidado e oferta de suporte social dentre muitos outros (ANESHENSEL, 1992; CFP, 2017; DAMASCENO; ZANELLO, 2018; SMOLEN; ARAUJO, 2019; TAVARES, 
KURATANI, 2019). No entanto, como afirmam Damasceno e Zanello (2018) este é um tema ainda pouco discutido no âmbito da saúde pública brasileira mesmo com alertas da Organização Mundial de Saúde.

Ignácio e Mattos (2019), analisando o processo do Grupo de Trabalho Racismo e Saúde Mental no Ministério da Saúde (GTRSM) que existiu no período de 2014 a 2016, nos informam que, apesar das indicações previstas na PNSIPN, o GTRSM pode ser considerado uma das únicas tentativas efetivas de tomar a saúde mental da população negra como uma questão relevante. Estes autores destacam as assimetrias raciais no campo da saúde mental, além de problemas na formação dos profissionais, a dificuldade da Rede de Atenção Psicossocial (RAPS) em acolher o sofrimento psíquico decorrente do racismo e escassez de linhas de cuidado que tenham como foco os sofrimentos causados pelo racismo e por discriminações raciais.

A RAPS representa um grande avanço no cuidado à saúde mental da população brasileira, é um outro exemplo de tentativa de ruptura com a violência manicomial, mas que tem fracassado no enfrentamento do racismo e passa por um processo acelerado de desmonte. Dentre os objetivos gerais da RAPS estão a ampliação do acesso à atenção psicossocial da população em geral; a promoção de vínculos das pessoas com transtornos mentais e com necessidades decorrentes do uso de crack, álcool e outras drogas e suas famílias aos pontos de atenção; a garantia da articulação e integração dos pontos de atenção das redes de saúde no território, qualificando o cuidado por meio do acolhimento, do acompanhamento contínuo e da atenção às urgências (BRASIL, 2018; UFMA, 2018). Para alcançar estes objetivos, a Rede de Saúde Mental deve incluir diversas ações e serviços, cabendo à Atenção Primária o papel de porta de entrada e ordenadora do cuidado. Além disso, entre os equipamentos que devem substituir os antigos hospitais psiquiátricos estão os Centros de Atenção Psicossocial (CAPS), os Serviços Residências Terapêuticos (SRT), os Centros de Convivência (Cecos), as Enfermarias de Saúde Mental em Hospitais Gerais, as oficinas de geração de renda, dentre outros (BRASIL, 2010).

Neste modelo, recomenda-se que o CAPS seja acionado apenas em casos que ultrapassem as possibilidades do cuidado conjunto entre Equipes de Saúde da Família e o Núcleo de Ampliado de Saúde da Família e Atenção Básica (NASF-AB), ou seja, casos mais graves (incluindo situações de crise) em que seja preciso o cuidado intensivo e/ou a

Revista da ABPN • v. 12, n. Ed. Especial - Caderno Temático: "III ANPSINEP Articulação Nacional de Psicólogas/os Negras/os e Pesquisadoras/es” • outubro de 


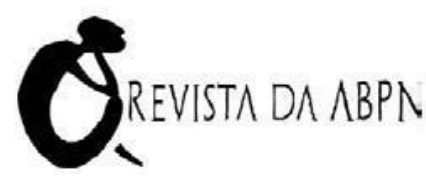

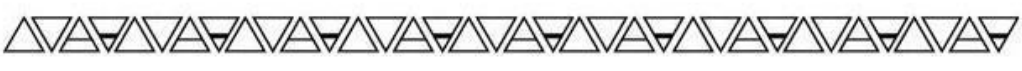

reinserção (UFMA, 2018). As Equipes de Saúde da Família e o NASF- AB cumprem uma importante função na assistência em saúde mental, criando, junto aos usuários, novas ferramentas e estratégias para compartilhar e construir o cuidado em saúde. Considera-se que mesmo "doenças físicas" trazem consigo uma porção de sofrimento associado às repercussões impostas por essas condições de saúde. Neste sentido, pode-se dizer que todo problema de saúde é também um problema de saúde mental, o que requer que as ESF estejam preparadas para acolher essas demandas (BRASIL, 2013b; BRASIL, 2010).

No contexto atual de retrocessos e desarticulação de políticas públicas resultantes de lutas sociais, a "nova" Política de saúde mental reinsere na Rede dois dispositivos que reproduzem a lógica manicomial - Hospitais Psiquiátricos e Comunidades Terapêuticas. Esta é uma forte ameaça à atenção da saúde mental da população negra uma vez que se perdem os avanços alcançados pelo movimento antimanicomial como a desospitalização e investimentos em ressocialização e seguimos, consistentemente, para o retorno ao modelo hegemônico do início do século XX.

Em nossa experiência no âmbito da RAPS, percebemos desconhecimento dos profissionais, especialmente da Atenção Primária à Saúde (APS), acerca da própria RAPS e da PNSIPN. Isto se reflete na desresponsabilização da APS em relação aos usuários em sofrimento mental e na escassez de discussões acerca de como o racismo poderia estar relacionado a estes quadros, tendo em vista que a maior parte dos usuários é negra.

Esta desresponsabilização, que afeta diretamente o vínculo dos usuários com as equipes, pode estar associada à expectativa (ou desejo) por parte dos profissionais da APS de que os serviços especializados assumam toda a demanda de saúde e crie estratégias de resolução dos problemas apresentados. Essa necessidade de transferir a responsabilidade pelos cuidados com a saúde mental dos usuários pode ter relação com psicofobia, que aparece em falas de profissionais e em situações de mau acolhimento ao usuário, assim como com o racismo institucional reconhecido na PNSIPN e outros documentos do Ministério da Saúde.

O racismo institucional no atendimento e cuidado à população negra na RAPS impede que o básico seja oferecido a estes usuários que representam mais de $70 \%$ dos que dependem exclusivamente do SUS. Ao não reconhecer e/ou não orientar suas ações para o enfrentamento do racismo e de suas repercussões, tornam-se inviáveis os processos

Revista da ABPN • v. 12, n. Ed. Especial - Caderno Temático: "III ANPSINEP Articulação Nacional de Psicólogas/os Negras/os e Pesquisadoras/es” • outubro de 2020, p. 138-151 
de vinculação, a escuta qualificada, a boa comunicação, ou seja, o modelo de cuidado preconizado pela Política Nacional de Saúde Mental.

Outro ponto que merece atenção nesta política e que deveria ter relação direta com medidas de enfrentamento do racismo como estratégia de promoção de saúde mental é a oferta de serviços no lócus onde os usuários vivenciam suas dimensões sociais, econômicas, políticas e culturais. A Política Nacional de Saúde Mental, a partir da categoria território, sugere um aporte político às intervenções em saúde mental no sentido de modificar e qualificar as condições e modos de vida, produzindo e promovendo saúde para além de um olhar único à doença. Aponta, ainda, que a construção dessas intervenções precisa se dar no cotidiano dos encontros entre o serviço e os assistidos com uma forte inserção territorial (BRASIL, 2013a).

O Ministério da Saúde reconhece que a situação de vulnerabilidade social afeta a saúde da população negra (BRASIL, 2013c) e que um dos marcadores de vulnerabilidade que podemos associar ao território são os altos índices de violência letal que afeta de forma desigual esta população (IPEA,2020). Souza e Lima (2006) indicam que os altos índices de violência urbana mantêm relação com a exclusão social e crime organizado, desemprego, desestruturação familiar e o sentimento de frustração diante de uma busca por padrões sociais mostrados como possíveis em um mundo consumista. Esses pontos se associam à população negra quando ressaltamos o processo histórico de segregação urbana, favelização e conseguinte criminalização das favelas, e o fato de que tais espaços são compostos majoritariamente por pessoas negras (IPEA, 2011).

\section{POR UMA POLÍTICA DE SAÚDE MENTAL RACIALIZADA NO SUS}

Ter uma política de saúde mental racializada é um alvo distante que exige luta política, organização social e persistência coletiva. Na fase em que estamos, ainda somos obrigadas a reinvindicar o básico e denunciar sistematicamente, por exemplo, que não temos acesso a informações de qualidade no SUS devido ao não preenchimento do quesito raça/cor nos sistemas de informação. Esta é uma questão crucial para produção e análise de dados epidemiológicos antes, durante a Pandemia de COVID-19 (SANTOS et al, 2020) e após, mas que só será superada com mudança significativa das prioridades e

Revista da ABPN • v. 12, n. Ed. Especial - Caderno Temático: "III ANPSINEP Articulação Nacional de Psicólogas/os Negras/os e Pesquisadoras/es” • outubro de 
investimentos governamentais, assim como com inclusão desta discussão na formação dos profissionais de saúde e construção coletiva de políticas públicas e outras ferramentas orientadoras e organizativas por meio da educação popular em saúde.

O enfrentamento do racismo no campo da saúde passa pela construção coletiva de um modelo assistencial que tenha em suas bases o letramento racial como potencializador das ações e no devido reconhecimento da importância dos saberes tradicionais e ancestrais produzidos pelos diversos grupo e comunidades negras.

Letramento racial é um conceito formulado pela antropóloga France Widdance Twine que fala sobre formas de respostas à tensões raciais buscando reeducar o indivíduo em uma perspectiva antirracista (SILVA, 2019). Dessa forma, esta estratégia pode orientar os usuários e profissionais de saúde sobre como identificar e responder às questões raciais de forma resolutiva.

Em relação ao reconhecimento dos saberes tradicionais e ancestrais, lembramos que atualmente são indicadas e adotadas práticas da Medicina Tradicional Chinesa (MTC) para o cuidado em saúde mental, considerando-se que estas ajudam a ampliar o olhar sobre o usuário sobre as desarmonias que levam ao sofrimento mental (BRASIL, 2013a). Contudo, não há menção sobre os cuidados que os povos tradicionais negros e indígenas sempre desenvolveram numa linha integral possivelmente mais adequada à população brasileira que MTC.

Podemos citar aqui muitas outras questões como se apropriar de epistemologias do Sul global, valorizar conhecimentos como a fitoterapia que tem suas origens apagadas e que são parte da produção de cuidados e memória da população negra brasileira. Racializar a RAPS significa caminhar em direção à terapêutica da ressubjetivação, colaborando com o processo de afirmar racial e positivamente o sujeito negro em busca do horizonte da justiça social (COSTA, 2015).

Pensar uma política pública que promova e recupere a saúde mental implica, portanto, em romper radicalmente com nossas heranças coloniais, com o racismo estrutural, com a lógica manicomial e garantir na prática os princípios fundamentais do SUS de universalidade, equidade e integralidade. Implica numa revisão profunda na formação dos profissionais de saúde e retorno às bases da educação em saúde que 
promova o controle social efetivo e que fomente o encontro respeitoso entre sujeitos e grupos diversos, com especial atenção às interseccionalidades que nos constituem

Concordando com Costa e Tavares (2020), consideramos que a luta antimanicomial e a antirracista são indissociáveis. Entendemos que, enquanto a organização de nossa sociedade for baseada em categorias como racismo, classismo, patriarcalismo e capacitismo, por exemplo, a saúde de grande parte da população estará em risco e teremos que atuar num modelo de redução de danos.

Portanto, é urgente a pactuação entre as diferentes esferas de governo de uma política de saúde mental racializada e que considere as necessidades da população negra atendida pelo SUS. E esta política será possível através da superação do racismo estrutural e, particularmente, de sua dimensão institucional, da necessária ruptura com o modelo manicomial atualizado nas comunidades terapêuticas e retorno ao modelo médico/ hospitalar/ privatista, da promoção de debate público sobre estas questões e, como defende Passos (2018, p. 14) através da implementação e efetivação da Reforma Psiquiátrica brasileira, pois "racializar os corpos e a própria história faz parte dos novos rumos da Luta Antimanicomial".

\section{REFERÊNCIAS BIBLIOGRÁFICAS}

ALMEIDA, S. O que é racismo estrutural? Belo Horizonte: Editora Letramento. 2018

ANESHENSEL, C. S. (1992). Social Stress: Theory and Research. Annual Review of Sociology, 18(1), 15-38.

AZEVEDO, Celia Maria Marinho de. Onda Negra, Medo Branco: O Negro no Imaginário das Elites do Século XIX. Rio de Janeiro, Paz e Terra, 1987.

BARRETO, L. Diário do Hospício / O Cemitério dos Vivos. $1^{\mathrm{a}}$ ed. São Paulo. Companhia das Letras. 2017.

BRASIL. Diretrizes do NASF: Núcleo de Apoio a Saúde da Família. 2010.160p.

BRASIL. MINISTÉRIO DA SAÚDE. Cadernos de Atenção Básica $n^{\circ} 34$ - Saúde Mental. Brasília, Editora MS, $1^{\text {a }}$ ed. 2013a

BRASIL. Ministério da Saúde. Secretaria de Atenção à Saúde. Departamento de Atenção Básica. Departamento de Ações Programáticas Estratégicas. Saúde Mental. Brasília: Ministério da Saúde, 2013b.

Revista da ABPN • v. 12, n. Ed. Especial - Caderno Temático: "III ANPSINEP Articulação Nacional de Psicólogas/os Negras/os e Pesquisadoras/es” • outubro de 
BRASIL. Ministério da Saúde. Secretaria de Gestão Estratégica e Participativa. Departamento de Apoio à Gestão Participativa. Política Nacional de Saúde Integral da População Negra. 2013c..35p.

BRASIL. Ministério da Saúde. Rede de Atenção Psicossocial. Disponível em: http://www.saude.gov.br/saude-para-voce/saude-mental/acoes-e-programas-saude-mental/redede-atencao-psicossocial-raps. Acesso em: 23 de set. de 2018.

CONSELHO FEDERAL DE PSICOLOGIA. Relações Raciais: Referências Técnicas para atuação de psicólogas/os. Brasília: CFP, 2017.

COSTA, Eliane Silvia. Racismo como metaenquadre. Rev. Inst. Estud. Bras., São Paulo, n. 62, p. 146-163, Dec. 2015.

COSTA, Eliane Silvia; TAVARES, Jeane Saskya Campos. A luta antimanicomial também é uma luta antirracista. In Boletim. Comissão de direitos humanos do CFP. [recurso eletronico]/ Conselho Federal de Psicologia. V.1, n1. Maio de 2020. Brasilia, 2020.

DAMASCENO, Marizete Gouveia; ZANELLO, Valeska M. Loyola. Saúde mental e racismo contra negros: produção bibliográfica brasileira dos últimos quinze anos. Psicologia: Ciência e Profissão, v. 38, n. 3, p. 450-464, 2018.

DAVIS, A. A liberdade é uma luta constante. São Paulo: Boitempo, 2018.

IGNÁCIO, Marcos Vinicius Marques; MATTOS, Ruben Araujo de. O Grupo de Trabalho Racismo e Saúde Mental do Ministério da Saúde: a saúde mental da população negra como questão. Saúde em Debate, v. 43, p. 66-78, 2019. Disponível em: $<$ https://www.scielo.br/scielo.php?script=sci_arttext\&pid=S010311042019001300066\&lng=en\&nrm=iso > Acesso em: 29 de set. de 2020.

INSTITUTO DE PESQUISA ECONÔMICA APLICADA - IPEA. Atlas da violência. Rio de Janeiro, RJ: o autor. 2020.

INSTITUTO DE PESQUISA ECONÔMICA APLICADA - IPEA. Retrato das Desigualdades de Gênero e Raça. $4^{\mathrm{a}}$ edição. IPEA: Brasília/DF, 2011.

PASSOS, R. G. "Holocausto ou Navio Negreiro?": inquietações para a Reforma Psiquiátrica brasileiro. Argumentum, 10(3), 10-23, 2018.2 Disponível em https://doi.org/10.18315/argumentum.v10i3.21483. Acesso em 28 de set de 2020

REIS, João José. Ganhadores: A greve negra de 1857 na Bahia. 1a ed. São Paulo: Companhia das Letras, 2019.

SANTOS, Marcia Pereira Alves dos et al . População negra e Covid-19: reflexões sobre racismo e saúde. Estud. av., São Paulo, v. 34, n. 99, p. 225-244, Aug. 2020. Disponível em: $<$ https://www.scielo.br/scielo.php?script=sci_arttext\&pid=S0103-

40142020000200225\&lng=en\&nrm=iso> . Acesso em: 30 set. 2020.

SERAPIONI, Mauro. Franco Basaglia: biografia de um revolucionário. Hist. cienc. SaúdeManguinhos, Rio de Janeiro, v. 26, n. 4, p. 1169-1187, Dec. 2019. Available from

Revista da ABPN • v. 12, n. Ed. Especial - Caderno Temático: "III ANPSINEP Articulação Nacional de Psicólogas/os Negras/os e Pesquisadoras/es” • outubro de 2020, p. 138-151 
$<$ https://www.scielo.br/scielo.php?script=sci_arttext\&pid=S0104-

59702019000401169\&lng=en\&nrm=iso>. Access on 29 Sept. 2020. Epub Nov 28, 2019. https://doi.org/10.1590/s0104-59702019000400008.

SILVA, Marcos Fabrício Lopes da. Educação e letramento racial. Boletim, Belo Horizonte, ano 46, n. 2081, p. 1-1, 18 nov. 2019. Disponível em: <https://ufmg.br/comunicacao/publicacoes/boletim/edicao/2081/educacao-e-letramento-racial> . Acesso em: 28 set. 2020.

SMOLEN, Jenny Rose, Araújo, Edna Maria de. (2017). Raça/cor da pele e transtornos mentais no Brasil: uma revisão sistemática. Ciência \& Saúde Coletiva, 22(12), 4021-4030.

SOUZA, Edinilsa Ramos de; LIMA, Maria Luiza Carvalho de. Panorama da violência urbana no Brasil e suas capitais. Ciênc. Saúde coletiva, Rio de Janeiro, v. 11, supl. p. 1211-1222, 2006.

SCHUCMAN, Lia Vainer. Racismo e antirracismo: a categoria raça em questão. Rev. psicol. polít, São Paulo, v. 10, n. 19, p. 41-55, jan. 2010. Disponível em http://pepsic.bvsalud.org/scielo.php?script=sci_arttext\&pid=S1519-

549X2010000100005\&lng=pt\&nrm=iso. Acesso em: 29 set. 2020.

TAVARES, Jeane Saskya Campos; KURATANI, Sayuri Miranda de Andrade. Manejo Clínico das Repercussões do Racismo entre Mulheres que se "Tornaram Negras". Psicol. Cienc. prof., Brasília, v.39, e184764, 2019 Epub 27-Jun-2019. https://doi.org/10.1590/1982-3703003184764.

UNIVERSIDADE FEDERAL DO MARANHÃO. Universidade Aberta do SUS. Curso de capacitação em Saúde Mental na Atenção Primária: Processos de trabalho colaborativo e as redes de atenção à saúde. São Luiz, MA: UFMA, 2018.

Recebido em: 18/09/2020

Aceito em: 01/10/2020

Revista da ABPN • v. 12, n. Ed. Especial - Caderno Temático: "III ANPSINEP Articulação Nacional de Psicólogas/os Negras/os e Pesquisadoras/es” • outubro de 2020, p. 138-151 